\title{
Interleukin-18 and Hematopoietic Recovery after Allogeneic Stem Cell Transplantation
}

\author{
Aleksandar Radujkovic ${ }^{1, *+\dagger}+\mathbb{C}$, Lambros Kordelas $2,+\left(\mathbb{D}\right.$, Rashit Bogdanov ${ }^{2}$, \\ Carsten Müller-Tidow ${ }^{1}$, Dietrich W. Beelen ${ }^{2}$, Peter Dreger ${ }^{1}$ and Thomas Luft ${ }^{1}$ \\ 1 Department of Internal Medicine V, University Hospital Heidelberg, 69120 Heidelberg, Germany; \\ Carsten.Mueller-Tidow@med.uni-heidelberg.de (C.M.-T.); Peter.Dreger@med.uni-heidelberg.de (P.D.); \\ Thomas.Luft@med.uni-heidelberg.de (T.L.) \\ 2 Department of Bone Marrow Transplantation, University Hospital Essen, University of Duisburg-Essen, \\ 45147 Essen, Germany; Lambros.Kordelas@uk-essen.de (L.K.); Rashit.Bogdanov@uk-essen.de (R.B.); \\ Dietrich.Beelen@uk-essen.de (D.W.B.) \\ * Correspondence: aleksandar.radujkovic@med.uni-heidelberg.de \\ + These authors contributed equally to this work.
}

Received: 4 September 2020; Accepted: 26 September 2020; Published: 28 September 2020

Simple Summary: We have previously shown that high pre-conditioning levels of Interleukin-18 were associated with worse survival after allogeneic stem cell transplantation due to increased non-relapse mortality. While no correlations with acute graft-versus-host disease were observed, interleukin-18-related excess mortality was mainly driven by fatal infectious complications. In multiple studies, delayed hematopoietic recovery and poor graft function following allogeneic stem cell transplantation has been demonstrated as a powerful predictor of non-relapse mortality. The present study links high interleukin-18 to delayed platelet recovery in allografted patients. Given the functions of interleukin-18 in regulating the quiescence of hematopoietic stem/progenitor cells, our findings may be explained by Interferon gamma-independent inhibitory effects of interleukin-18 on stem cell proliferation and hematopoietic reconstitution in allografted patients. Importantly, considering recent successful interleukin-18-neutralizing approaches in autoimmune disorders, our results provide a rationale to explore modulation of interleukin-18 for improving hematopoietic recovery and outcomes in allogeneic stem cell transplantation recipients.

\begin{abstract}
Interleukin-18 (IL-18) is an immunoregulatory cytokine and a context-dependent regulator of hematopoietic stem/progenitor cell (HSPC) quiescence in murine models. In a previous study, high pre-conditioning levels of IL-18 were associated with increased non-relapse mortality (NRM) after allogeneic stem cell transplantation (alloSCT). To investigate the clinical impact of IL-18 status on hematopoietic function, the associations of pre-conditioning and day 0-3 cytokine levels with platelet and neutrophil recovery were analyzed in a training cohort of 714 allografted patients. In adjusted logistic regression analyses, both increasing pre-conditioning and day 0-3 IL-18 levels had a significantly higher adjusted odds ratio (aOR) of delayed platelet and neutrophil recovery on day +28 post-transplant (aOR per two-fold increase: 1.6-2.0). The adverse impact of high pre-conditioning IL-18 on day +28 platelet recovery was verified in an independent cohort of 673 allografted patients (aOR per two-fold increase: 1.8 and 1.7 for total and free IL-18, respectively). In both cohorts, a platelet count $\leq 20 / \mathrm{nL}$ on day +28 was associated with a significantly increased hazard of NRM (hazard ratio 2.13 and 2.94, respectively). Our findings support the hypothesis that elevated peritransplant IL-18 levels affect post-transplant HSPC function and may provide a rationale to explore modulation of IL-18 for improving alloSCT outcomes.
\end{abstract}


Keywords: interleukin-18; hematopoietic recovery; allogeneic stem cell transplantation; non-relapse mortality

\section{Introduction}

Allogeneic stem cell transplantation (alloSCT) is an established curative approach for a wide array of malignant and non-malignant hematologic disorders. However, besides persistence or recurrence of the primary disease, several complications can occur, including hemorrhages, infections, graft-versushost disease (GVHD), and endothelial damage-related toxicities, translating into considerable treatmentassociated morbidity and mortality.

Robust recovery and reconstitution of a donor-derived hematopoietic system in the recipient is of key importance for successful alloSCT. Accordingly, delayed hematopoietic recovery and poor graft function are associated with unfavorable outcomes [1-4]. In particular, delayed platelet recovery has been demonstrated to be a powerful predictor of increased non-relapse mortality (NRM) [2,5-9].

Interleukin-18 (IL-18) is a pro-inflammatory, interferon (IFN) $\gamma$-inducing, immunoregulatory cytokine involved in both innate and adaptive immune responses [10-12]. The IL-18 precursor is constitutively expressed in nearly all cells in humans including endothelial cells [10]. Maturation and secretion of IL-18 is mediated by the inflammasome [12], and its activity is inhibited by high-affinity binding with IL-18 binding protein (IL-18BP) [10,11].

In clinical settings, increased levels of IL-18 have been associated with a variety of autoimmune disorders including GVHD [11,13]. In addition, being part of the human host defense against infection, elevated IL-18 was reported to correlate with infection severity and outcome [14,15]. Accordingly, higher pre-conditioning levels of free IL-18 were found to be associated with non-relapse and overall mortality after alloSCT [16]. While no correlations with acute GVHD were observed, IL-18-related excess mortality was mainly driven by fatal infectious complications.

Using proximity-based differential single-cell analysis of the bone marrow (BM) niche Silberstein et al. [17] identified a previously unrecognized function of IL-18 as a regulator of hematopoietic stem/progenitor cells (HSPC) quiescence. In murine transplant models, IL-18 protected HSPC from injury caused by exposure to the cell-cycle-specific genotoxin 5-fluorouracil (5-FU), but in doing so, limited HSPC proliferation. In the clinical context of alloSCT, these findings may suggest a role of IL-18 in regulating post-transplant hematopoietic recovery and homeostasis.

IL-18 induces IFN $\gamma$, and IFN $\gamma$ conversely increases the gene expression and synthesis of both IL-18BP and several IFN $\gamma$-inducible CXC family chemokines. However, it should be noted that the BM niche factor function of IL-18 regulating HSPC quiescence is likely to involve IFN $\gamma$-independent effects of IL-18 [17], and therapeutic modulation of the IL-18 pathway is considered to be more effective in conditions with a minor role for IFN $\gamma$ [18].

In light of the above considerations, in the present study, we sought to investigate a possible link between IL-18 status and delayed post-transplant hematopoietic reconstitution focusing on IL-18, IL-18BP, IFN $\gamma$ and the IFN $\gamma$-inducible CXC family chemokines CXCL9 and CXCL10.

\section{Results}

\subsection{Patient Characteristics and Cytokine Serum Levels}

Patient characteristics of both cohorts are summarized in Table 1 . There were more patients with late stage and lymphoid disease in the training cohort. In the confirmation cohort, fewer patients had received reduced intensity conditioning, ATG in-vivo T-cell depletion and mycophenolate mofetil-containing GVHD prophylaxis. Baseline cytokine levels of both cohorts are also given in Table 1. Levels of total IL-18 and IL-18BP were higher in the confirmation cohort, whereas levels of free IL-18 were similar in both cohorts. 
Table 1. Baseline patient characteristics and cytokine serum levels.

\begin{tabular}{|c|c|c|c|}
\hline Parameter & $\begin{array}{l}\text { Training Cohort } \\
\quad(n=714)\end{array}$ & $\begin{array}{l}\text { Confirmation Cohort } \\
\qquad(n=673)\end{array}$ & $p$ \\
\hline $\begin{array}{l}\text { Age (years) at alloSCT } \\
\text { (median, IQR) }\end{array}$ & $54(45-61)$ & $54(44-61)$ & 0.71 \\
\hline $\begin{array}{l}\text { Patient sex, } n(\%) \\
\text { Female } \\
\text { Male }\end{array}$ & $\begin{array}{l}274(38) \\
440(62)\end{array}$ & $\begin{array}{l}314(47) \\
359(53)\end{array}$ & 0.002 \\
\hline $\begin{array}{c}\text { Disease stage } \\
\text { before alloSCT }{ }^{\mathrm{a}}, n(\%) \\
\text { Early } \\
\text { Intermediate } \\
\text { Late }\end{array}$ & $\begin{array}{l}251(35) \\
204(29) \\
259(36)\end{array}$ & $\begin{array}{l}304(45) \\
235(35) \\
134(20)\end{array}$ & $<0.001$ \\
\hline $\begin{array}{c}\text { Diagnosis, } n(\%) \\
\text { AML } \\
\text { MDS/MPN } \\
\text { Lymphoma } \\
\text { ALL } \\
\text { MM }\end{array}$ & $\begin{array}{l}261(37) \\
121(17) \\
222(31) \\
28(4) \\
82(11) \\
\end{array}$ & $\begin{array}{l}344(51) \\
134(20) \\
99(15) \\
70(10) \\
26(4)\end{array}$ & $<0.001$ \\
\hline $\begin{array}{c}\text { Conditioning }^{\mathrm{b}}, n(\%) \\
\text { RIC } \\
\text { MAC }\end{array}$ & $\begin{array}{c}649(91) \\
65(9)\end{array}$ & $\begin{array}{l}531(79) \\
142(21)\end{array}$ & $<0.001$ \\
\hline $\begin{array}{c}\text { Donor, } n(\%) \\
\text { RD } \\
\text { MUD } \\
\text { MMUD }\end{array}$ & $\begin{array}{l}207(29) \\
359(50) \\
148(21)\end{array}$ & $\begin{array}{l}192(29) \\
319(47) \\
162(24)\end{array}$ & 0.31 \\
\hline $\begin{array}{l}\text { Donor sex, } n(\%) \\
\text { Female } \\
\text { Male }\end{array}$ & $\begin{array}{l}229(32) \\
485(68)\end{array}$ & $\begin{array}{l}232(34) \\
441(66)\end{array}$ & 0.34 \\
\hline $\begin{array}{c}\text { ATG treatment, } n(\%) \\
\text { No } \\
\text { Yes }\end{array}$ & $\begin{array}{l}222(31) \\
492(69)\end{array}$ & $\begin{array}{l}284(42) \\
389(58)\end{array}$ & $<0.001$ \\
\hline $\begin{array}{c}\text { GVHD prophylaxis, } n(\%) \\
\text { CNI + MTX } \\
\text { CNI + MMF }\end{array}$ & $\begin{array}{l}216(30) \\
498(70) \\
\end{array}$ & $\begin{array}{c}632(94) \\
41(6)\end{array}$ & $<0.001$ \\
\hline $\begin{array}{c}\text { Stem cell source, } n(\%) \\
\text { Peripheral blood } \\
\text { Bone marrow }\end{array}$ & $\begin{array}{c}673(94) \\
41(6)\end{array}$ & $\begin{array}{l}603(90) \\
70(10)\end{array}$ & 0.001 \\
\hline $\begin{array}{l}\text { Pre-conditioning total IL-18 } \\
\quad(\mathrm{pg} / \mathrm{mL}) \text { (median, IQR) }\end{array}$ & $627(437-930)$ & $692(462-1026)$ & 0.04 \\
\hline $\begin{array}{c}\text { Pre-conditioning IL-18BP } \\
\text { (pg/mL) (median, IQR) }\end{array}$ & $5200(4313-5987)$ & $11,404(9282-12,101)$ & $<0.001$ \\
\hline $\begin{array}{l}\text { Pre-conditioning free IL-18 } \\
\text { (pg/mL) (median, IQR) }\end{array}$ & $414(266-658)$ & $414(283-608)$ & 0.96 \\
\hline $\begin{array}{l}\text { Pre-conditioning CXCL9 } \\
(\mathrm{pg} / \mathrm{mL}) \text { (median, IQR) }\end{array}$ & 199 (85-679) & $211(114-382)$ & 0.92 \\
\hline $\begin{array}{l}\text { Pre-conditioning CXCL10 } \\
\text { (pg/mL) (median, IQR) }\end{array}$ & $85(45-186)$ & - & - \\
\hline $\begin{array}{l}\text { Pre-conditioning IFN } \gamma \\
(\mathrm{pg} / \mathrm{mL})(\text { median, IQR) }\end{array}$ & $7.5(2.0-18.7)$ & - & - \\
\hline
\end{tabular}

Abbreviations: ALL, acute lymphoblastic leukemia; alloSCT, allogeneic stem cell transplantation; AML, acute myeloid leukemia; ATG, anti-thymocyte globulin; CNI, calcineurin inhibitor; $\mathrm{CXCL}$, chemokine (C-X-C motif) ligand; IFN $\gamma$, interferon gamma; IL-18, interleukin-18; IL-18BP, interleukin-18 binding protein; IQR, interquartile range; HLA, human leukocyte antigen; MAC, myeloablative conditioning; MDS, myelodysplastic syndrome; MM, multiple myeloma; MMF, mycophenolate mofetil; MMUD, mismatched unrelated donor; MPN, myeloproliferative neoplasm; MTX, methotrexate; MUD, matched unrelated donor; RD, related donor; RIC, reduced intensity conditioning.

a According to Gratwohl et al. [19]. ${ }^{\text {b }}$ According to Bacigalupo et al. [20] and Bornhäuser et al. [21]. 
Demographics, disease and transplant characteristics of the subgroup of patients who had serum samples available at day 0-3 peri-transplant $(n=306)$ were comparable to that of the entire training cohort Table S1. Pre-conditioning serum levels of total IL-18, IL-18BP as well as free IL-18 were lower than the levels observed at day 0-3 peri-transplant. Levels of IL-18 levels (total and free) were positively correlated (Table S2).

Pre-conditioning and day 0-3 IL-18 levels were not associated with patient age, patient gender, the hematopoietic cell transplantation-specific comorbidity index (HCT-CI) and the disease stage prior to alloSCT in the training cohort. Pre-conditioning total and free IL-18 levels were increased in patients with higher C-reactive protein (CRP) and ferritin levels prior to the start of the conditioning. For day 0-3 total and free IL-18 levels only a trend towards increased levels in patients with higher pre-conditioning CRP levels was observed, whereas pre-conditioning ferritin levels were not associated with day 0-3 IL-18 (Figures S1-S4).

\subsection{Platelets and ANC in Patients with Low Versus High IL-18 Levels}

Platelet and neutrophil counts were evaluated with regard to both total and free IL-18 in the present study. High levels of pre-conditioning total and free IL-18 (as defined by the fourth quartile (Q4) of the corresponding distribution) were associated with significantly lower median platelet counts as compared to low IL-18 levels (Q1) at all time-points investigated including the pre-conditioning landmark (Figure 1A,C). The differences in median platelet counts in patients with low (Q1) versus high (Q4) pre-conditioning IL-18 levels diminished with increasing time from transplant. No significant associations of IL-18 levels with regard to ANC were observed (Figure 1B,D).

Similar associations were observed for day $0-3$ total and free IL-18 and platelet counts (Figure 1E,G). In addition, high day 0-3 IL-18 levels were also correlated to lower median neutrophil counts at day +28 and day +50 post-transplant (Figure $1 \mathrm{~F}, \mathrm{H}$ ).

In the confirmation cohort, patients with high pre-conditioning levels of total and free IL-18 had significantly lower median platelet counts both before alloSCT and at day +28 post-transplant (Figure 2A,C), whereas higher levels of pre-conditioning total and free IL-18 were associated with lower median ANC on day +28 and prior to alloSCT, respectively (Figure 2B,D).

The corresponding Spearman's rank correlation coefficients for both cohorts were summarized in Table S3.

\subsection{Cytokine Serum Levels and Hematopoietic Recovery}

In the training cohort, higher pre-conditioning total and free (continuous) IL-18 levels predicted non-achievement of defined platelet and neutrophil recovery milestones (Table 2). As per area under the ROC curve, the most informative cytokine for predicting delayed platelet recovery on day +28 was free IL-18. In contrast, no associations of platelet and neutrophil recovery with pre-conditioning levels of IL-18BP, IFN $\gamma$ and the IFN $\gamma$-inducible chemokines CXCL9 and CXCL10 were observed (Table 2). For day 0-3 IL-18 levels, similar and stronger associations were found in univariate analysis particularly for day +28 platelet and neutrophil recovery on $(\mathrm{OR} \geq 2)$ (Table 3$)$. In addition, a correlation between delayed platelet recovery and higher levels of IL-18BP and IFN $\gamma$ at day $0-3$ was observed but only for the day +50 milestone. 

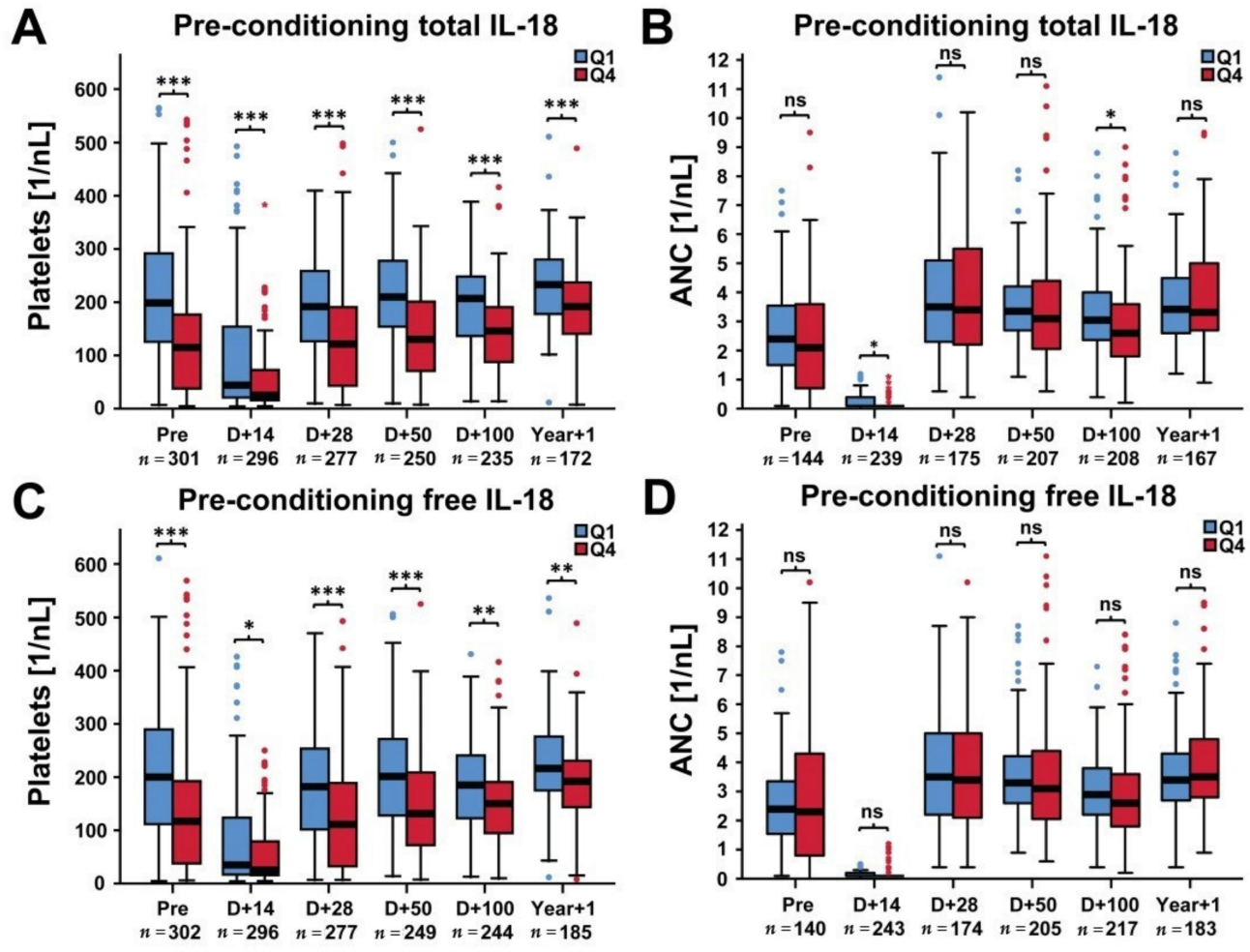

D

Pre-conditioning free IL-18
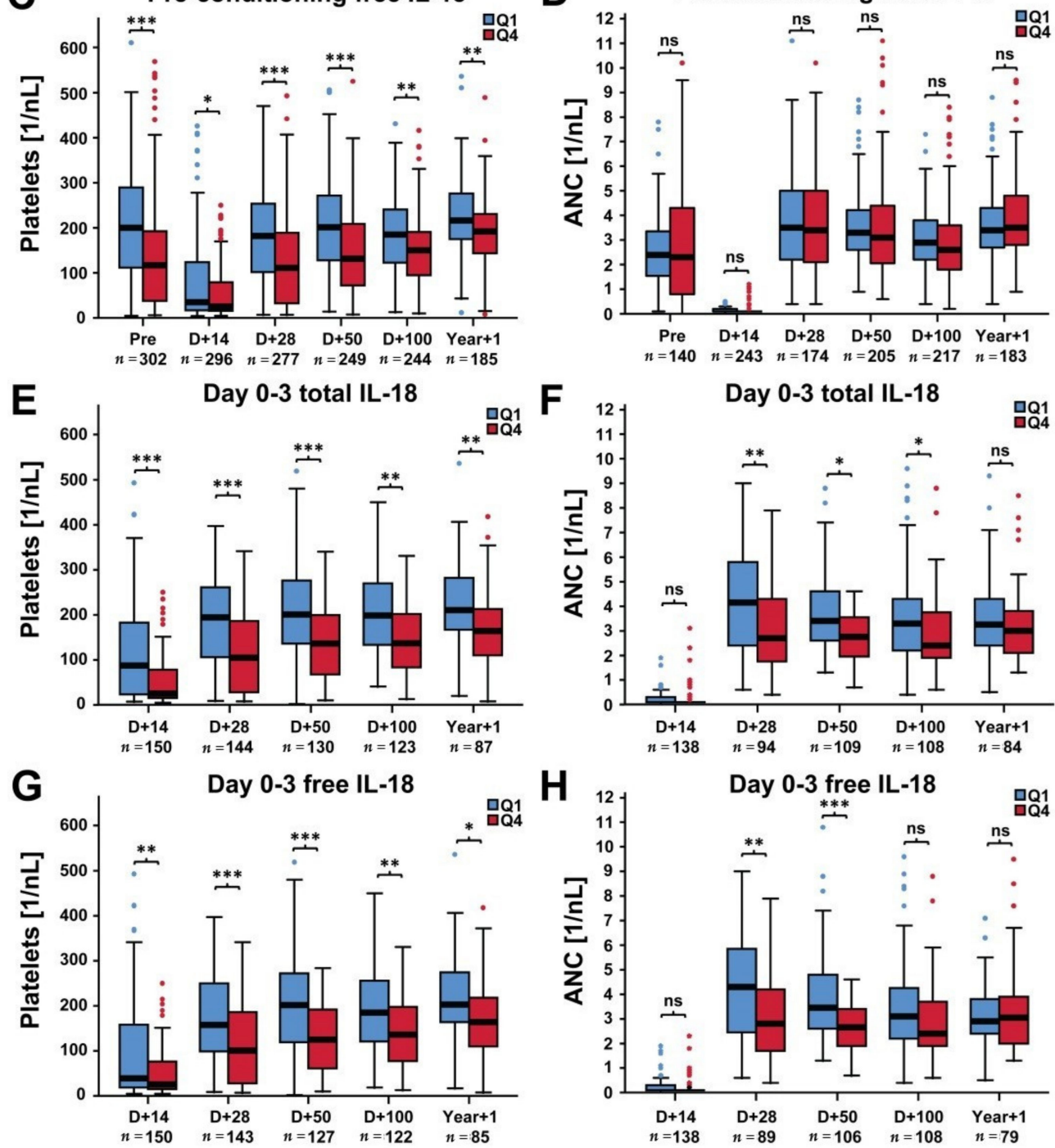

Figure 1. Median platelet and neutrophil counts pre-conditioning and at different time-points following alloSCT in patients with low versus high pre-conditioning and day 0-3 total and free IL-18 levels (training cohort). (A,B) Median platelet and neutrophil counts, respectively, in patients with low versus high pre-conditioning total IL-18 levels. (C,D) Median platelet and neutrophil counts, respectively, according to pre-conditioning free IL-18 levels. (E,F) Median platelet and neutrophil counts, respectively, in patients with low versus high day 0-3 total IL-18 levels. (G,H) Median platelet and neutrophil counts, respectively, according to day 0-3 free IL-18 levels. The first (Q1) and fourth quartile (Q4) of the total IL-18 and free IL-18 distribution was used to stratify patients in low and high IL-18 groups. Corresponding patient numbers are given $(n=\mathrm{Q} 1+\mathrm{Q} 4)$. Mann-Whitney U test, ${ }^{*} p<0.05,{ }^{* *} p<0.01,{ }^{* * *} p<0.001, \mathrm{~ns}=$ not significant. 
A Pre-conditioning total IL-18

B Pre-conditioning total IL-18
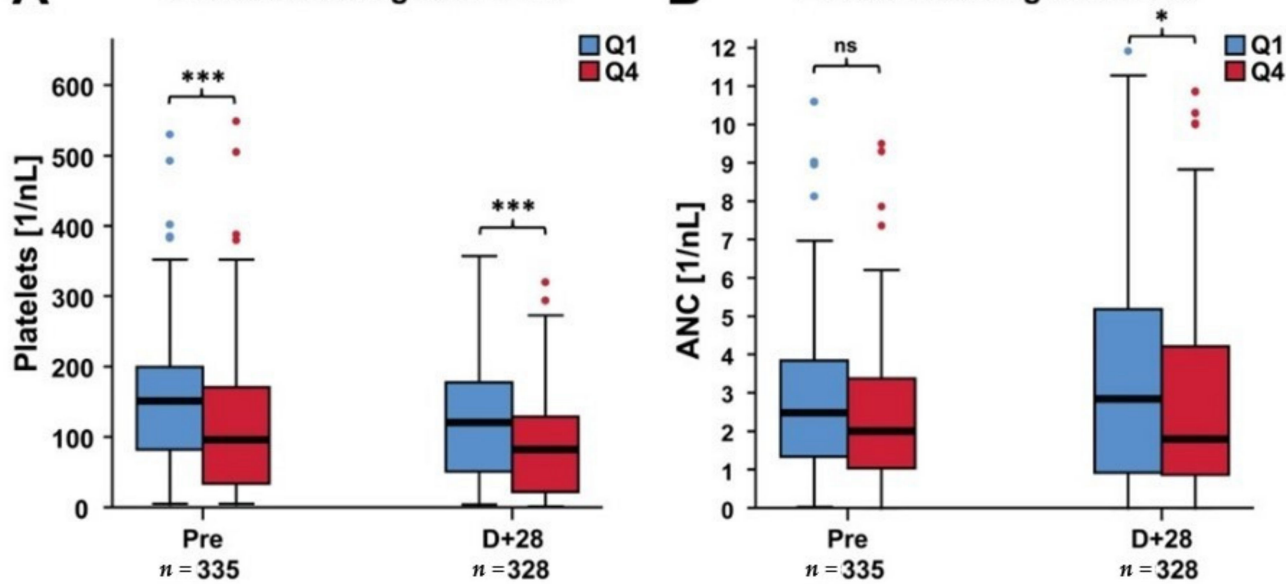

C Pre-conditioning free IL-18

D Pre-conditioning free IL-18
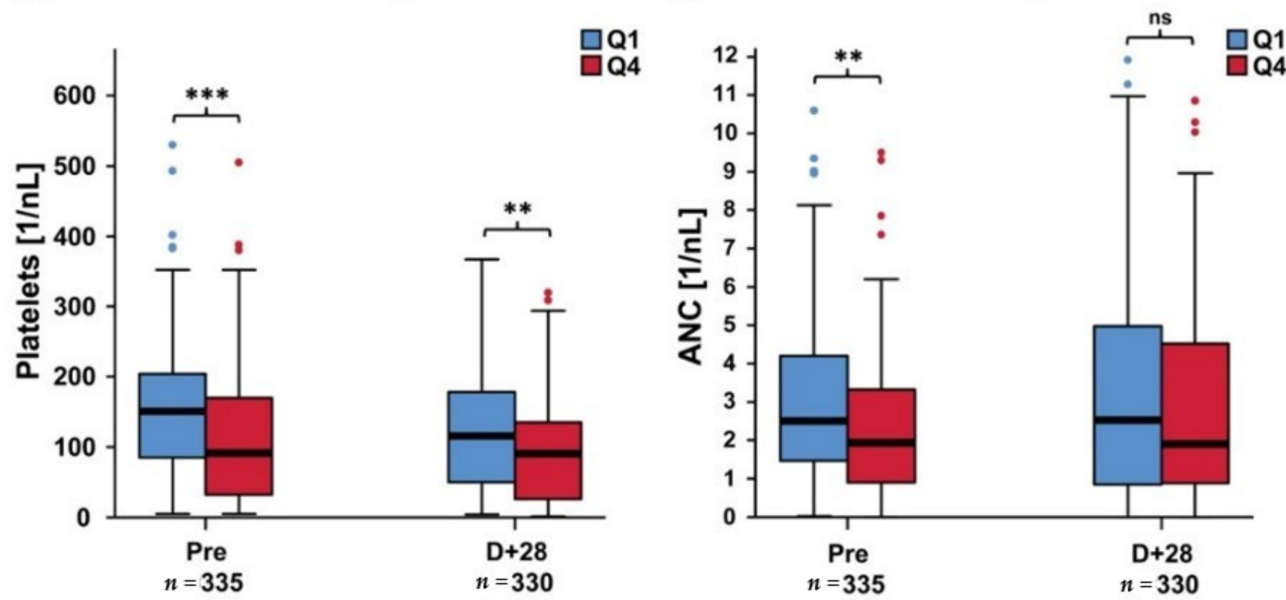

Figure 2. Median platelet and neutrophil counts pre-conditioning and at day +28 following alloSCT in patients with low versus high pre-conditioning total and free IL-18 levels (confirmation cohort). (A,B) Median platelet and neutrophil counts, respectively, in patients with low versus high pre-conditioning total IL-18 levels. (C,D) Median platelet and neutrophil counts, respectively, according to pre-conditioning free IL-18 levels. The first (Q1) and fourth quartile (Q4) of the total IL-18 and free IL-18 distribution was used to stratify patients in low and high IL-18 groups. Corresponding patient numbers are given ( $n=\mathrm{Q} 1+\mathrm{Q} 4)$. Mann-Whitney U test, ${ }^{*} p<0.05,{ }^{* *} p<0.01,{ }^{* * *} p<0.001$, ns $=$ not significant. 
Table 2. Associations of pre-conditioning cytokine serum levels with platelet and neutrophil recovery and corresponding predictive values (training cohort).

\begin{tabular}{|c|c|c|c|c|}
\hline \multirow[b]{2}{*}{ Cytokine } & \multicolumn{4}{|c|}{ Odds Ratio (OR) and Area under the ROC Curve (AUC) per log2 Increase * in Pre-Conditioning Cytokine } \\
\hline & $\begin{array}{c}\text { Platelets Day }+28 \leq 20 / \mathrm{nL} \\
(n=41) \text { vs. }>20 / \mathrm{nL}(n=512)\end{array}$ & $\begin{array}{c}\text { Platelets Day }+50 \leq 100 / \mathrm{nL} \\
(n=117) \text { vs. }>100 / \mathrm{nL}(n=391)\end{array}$ & $\begin{array}{c}\text { Platelets Year }+1 \leq 150 / \mathrm{nL} \\
(n=84) \text { vs. }>150 / \mathrm{nL}(n=274)\end{array}$ & $\begin{array}{c}\text { ANC Day }+28 \leq 1 / \mathrm{nL} \\
(n=43) \mathrm{vs} \\
>1 / \mathrm{nL}(n=331)\end{array}$ \\
\hline \multicolumn{5}{|l|}{ Total IL-18 } \\
\hline OR $(95 \% \mathrm{CI}), p$ & $1.56(1.20-2.03), 0.001$ & $1.52(1.24-1.86),<0.001$ & $1.28(1.01-1.61), 0.04$ & $1.61(1.23-2.13), 0.001$ \\
\hline $\operatorname{AUC}(95 \% \mathrm{CI}), p$ & $0.68(0.60-0.76),<0.001$ & $0.63(0.58-0.69),<0.001$ & $0.57(0.50-0.64), 0.04$ & $0.65(0.56-0.74), 0.002$ \\
\hline \multicolumn{5}{|l|}{ IL-18BP } \\
\hline OR $(95 \% \mathrm{CI}), p$ & $0.78(0.60-1.02), 0.07$ & $0.98(0.86-1.11), 0.72$ & $0.96(0.82-1.10), 0.55$ & $0.86(0.68-1.09), 0.20$ \\
\hline $\operatorname{AUC}(95 \% \mathrm{CI}), p$ & $0.46(0.38-0.54), 0.41$ & $0.50(0.45-0.56), 0.94$ & $0.51(0.44-0.58), 0.75$ & $0.50(0.41-0.58), 0.91$ \\
\hline \multicolumn{5}{|l|}{ Free IL-18 } \\
\hline OR $(95 \%$ CI $), p$ & $1.72(1.32-2.23),<0.001$ & $1.45(1.20-1.75),<0.001$ & $1.23(1.01-1.50), 0.04$ & $1.81(1.36-2.41),<0.001$ \\
\hline $\operatorname{AUC}(95 \% \mathrm{CI}), p$ & $0.72(0.65-0.79),<0.001$ & $0.64(0.58-0.69),<0.001$ & $0.56(0.49-0.63), 0.12$ & $0.67(0.58-0.76),<0.001$ \\
\hline \multicolumn{5}{|l|}{ CXCL9 } \\
\hline OR $(95 \%$ CI), $p$ & $0.93(0.81-1.06), 0.27$ & 0.99 (0.91-1.09), 0.91 & $0.98(0.88-1.08), 0.62$ & $1.02(0.88-1.17), 0.80$ \\
\hline $\operatorname{AUC}(95 \% \mathrm{CI}), p$ & $0.43(0.34-0.52), 0.13$ & $0.50(0.44-0.56), 0.96$ & $0.47(0.41-0.54), 0.42$ & $0.50(0.42-0.58), 0.98$ \\
\hline \multicolumn{5}{|l|}{ CXCL10 } \\
\hline OR $(95 \% \mathrm{CI}), p$ & $1.01(0.87-1.18), 0.90$ & $0.98(0.88-1.08), 0.63$ & $0.95(0.85-1.06), 0.38$ & $1.02(0.87-1.18), 0.83$ \\
\hline $\operatorname{AUC}(95 \% \mathrm{CI}), p$ & $0.50(0.42-0.59), 0.93$ & $0.47(0.41-0.53), 0.39$ & $0.47(0.40-0.53), 0.36$ & $0.50(0.41-0.59), 0.96$ \\
\hline \multicolumn{5}{|l|}{$\mathrm{IFN} \gamma$} \\
\hline OR $(95 \% \mathrm{CI}), p$ & $1.06(0.91-1.25), 0.44$ & $1.01(0.91-1.12), 0.86$ & $0.90(0.79-1.01), 0.08$ & 1.07 (0.91-1.27), 0.39 \\
\hline AUC $(95 \% \mathrm{CI}), p$ & $0.54(0.44-0.65), 0.39$ & $0.50(0.44-0.57), 0.94$ & $0.43(0.36-0.50), 0.05$ & $0.48(0.39-0.57), 0.55$ \\
\hline
\end{tabular}


Table 3. Associations of day 0-3 cytokine serum levels with platelet and neutrophil recovery and corresponding predictive values (training cohort).

\begin{tabular}{|c|c|c|c|c|}
\hline \multirow[b]{2}{*}{ Cytokine } & \multicolumn{4}{|c|}{ Odds Ratio (OR) and Area under the ROC Curve (AUC) per log2 Increase * in Day 0-3 Cytokine } \\
\hline & $\begin{array}{c}\text { Platelets Day }+28 \leq 20 / \mathrm{nL} \\
(n=28) \text { vs. }>20 / \mathrm{nL}(n=257)\end{array}$ & $\begin{array}{c}\text { Platelets Day }+50 \leq 100 / \mathrm{nL} \\
(n=67) \text { vs. }>100 / \mathrm{nL}(n=196)\end{array}$ & $\begin{array}{c}\text { Platelets Year }+1 \leq 150 / \mathrm{nL} \\
(n=55) \text { vs. }>150 / \mathrm{nL}(n=125)\end{array}$ & $\begin{array}{c}\text { ANC Day }+28 \leq 1 / \mathrm{nL} \\
(n=31) \text { vs. }>1 / \mathrm{nL}(n=175)\end{array}$ \\
\hline \multicolumn{5}{|l|}{ Total IL-18 } \\
\hline OR $(95 \% \mathrm{CI}), p$ & $2.27(1.52-3.39),<0.001$ & $1.95(1.40-2.72),<0.001$ & $1.72(1.19-2.48), 0.004$ & $2.07(1.34-3.21), 0.001$ \\
\hline $\operatorname{AUC}(95 \% \mathrm{CI}), p$ & $0.70(0.59-0.81), 0.001$ & $0.68(0.61-0.75),<0.001$ & $0.63(0.55-0.72), 0.005$ & $0.67(0.56-0.79), 0.002$ \\
\hline \multicolumn{5}{|l|}{ IL-18BP } \\
\hline OR $(95 \% \mathrm{CI}), p$ & $1.50(0.76-2.96), 0.29$ & $1.70(1.02-2.84), 0.04$ & $1.03(0.62-1.68), 0.92$ & $1.45(0.75-2.78), 0.27$ \\
\hline $\operatorname{AUC}(95 \% \mathrm{CI}), p$ & $0.55(0.45-0.66), 0.35$ & $0.59(0.52-0.66), 0.03$ & $0.49(0.40-0.58), 0.82$ & $0.58(0.48-0.68), 0.15$ \\
\hline \multicolumn{5}{|l|}{ Free IL-18 } \\
\hline OR $(95 \% \mathrm{CI}), p$ & $2.12(1.45-3.10),<0.001$ & $1.86(1.34-2.59),<0.001$ & $1.74(1.21-2.48), 0.003$ & 1.95 (1.28-2.96), 0.002 \\
\hline $\operatorname{AUC}(95 \% \mathrm{CI}), p$ & $0.70(0.58-0.81), 0.001$ & $0.68(0.61-0.75),<0.001$ & $0.64(0.56-0.73), 0.002$ & $0.66(0.55-0.78), 0.004$ \\
\hline \multicolumn{5}{|l|}{ CXCL9 } \\
\hline OR $(95 \% \mathrm{CI}), p$ & $1.21(1.01-1.46), 0.04$ & 1.09 (0.97-1.21), 0.14 & $0.98(0.86-1.12), 0.77$ & $1.05(0.89-1.23), 0.58$ \\
\hline $\operatorname{AUC}(95 \% \mathrm{CI}), p$ & $0.61(0.49-0.72), 0.06$ & $0.56(0.47-0.64), 0.18$ & $0.47(0.38-0.57), 0.56$ & $0.54(0.42-0.66), 0.49$ \\
\hline \multicolumn{5}{|l|}{$\mathrm{IFN} \gamma$} \\
\hline OR (95\% CI), $p$ & 1.20 (0.99-1.44), 0.06 & 1.29 (1.11-1.51), 0.001 & 1.01 (0.85-1.19), 0.93 & $1.20(0.99-1.46), 0.06$ \\
\hline $\operatorname{AUC}(95 \% \mathrm{CI}), p$ & $0.61(0.50-0.71), 0.07$ & $0.66(0.58-0.74),<0.001$ & $0.52(0.42-0.62), 0.72$ & $0.64(0.53-0.75), 0.02$ \\
\hline
\end{tabular}

* Each one unit increase in log2 corresponds to a doubling in the corresponding cytokine level. Abbreviations: ANC, absolute neutrophil count; AUC, area under the ROC curve; $\mathrm{CI}$, confidence interval; CXCL, chemokine (C-X-C motif) ligand; IFN $\gamma$, interferon gamma; IL-18, interleukin-18; IL-18BP, interleukin-18 binding protein; OR, odds ratio; ROC, receiver operating characteristic. 
In multivariable analyses adjusting for donor match, graft source, ATG use, conditioning intensity, and disease score, increasing pre-conditioning IL-18 levels (total and free) were significantly associated with missing the day +28 platelet and neutrophil milestones (adjusted OR per two-fold increase 1.6-1.8, Table 4). Multivariable models including day 0-3 IL-18 serum levels yielded similar and more pronounced associations (adjusted OR per two-fold increase 1.7-2.0, Table S4). 
Table 4. Multivariable logistic regression analysis of pre-conditioning total and free IL-18 serum levels with regard to platelet and neutrophil recovery (training cohort).

\begin{tabular}{|c|c|c|c|c|c|c|c|c|}
\hline \multirow[b]{2}{*}{ Covariate, Effect } & \multicolumn{2}{|c|}{$\begin{array}{c}\text { Platelets Day }+28 \leq 20 / \mathrm{nL}(n=41) \text { vs. }>20 / \mathrm{nL} \\
(n=512)\end{array}$} & \multicolumn{2}{|c|}{$\begin{array}{c}\text { Platelets Day }+50 \leq 100 / \mathrm{nL}(n=117) \text { vs. } \\
>100 / \mathrm{nL}(n=391)\end{array}$} & \multicolumn{2}{|c|}{$\begin{array}{c}\text { Platelets Year }+1 \leq 150 / \mathrm{nL}(n=84) \text { vs. } \\
>150 / \mathrm{nL}(n=274)\end{array}$} & \multicolumn{2}{|c|}{$\begin{array}{c}\text { ANC Day }+28 \leq 1 / \mathrm{nL} \\
(n=43) \text { vs. }>1 / \mathrm{nL}(n=331)\end{array}$} \\
\hline & $\begin{array}{l}\text { Model with total } \\
\text { IL-18, } \\
\text { aOR }(95 \% \text { CI), } p\end{array}$ & $\begin{array}{c}\text { Model with } \\
\text { free IL-18, } \\
\text { aOR }(95 \% \text { CI }), p\end{array}$ & $\begin{array}{l}\text { Model with total } \\
\text { IL-18, } \\
\text { aOR }(95 \% \text { CI), } p\end{array}$ & $\begin{array}{c}\text { Model with } \\
\text { free IL-18, } \\
\text { aOR }(95 \% \text { CI), } p\end{array}$ & $\begin{array}{l}\text { Model with total } \\
\text { IL-18, } \\
\text { aOR }(95 \% \text { CI), } p\end{array}$ & $\begin{array}{c}\text { Model with } \\
\text { free IL-18, } \\
\text { aOR }(95 \% \text { CI), } p\end{array}$ & $\begin{array}{l}\text { Model with total } \\
\text { IL-18, } \\
\text { aOR }(95 \% \text { CI), } p\end{array}$ & $\begin{array}{c}\text { Model with } \\
\text { free IL-18, } \\
\text { aOR }(95 \% \text { CI), } p\end{array}$ \\
\hline $\begin{array}{c}\text { Total IL-18, per log2 } \\
\text { increase * }\end{array}$ & 1.61 (1.21-2.15), 0.001 & - & $\begin{array}{c}1.52(1.22-1.88) \\
<0.001\end{array}$ & - & $1.28(1.01-1.63), 0.04$ & - & $1.63(1.20-2.21), 0.002$ & - \\
\hline $\begin{array}{c}\text { Free IL-18, per log2 } \\
\text { increase * }\end{array}$ & - & $\begin{array}{c}1.78(1.34-2.36) \\
<0.001\end{array}$ & - & $1.43(1.18-1.74), 0.002$ & - & $1.22(0.99-1.50), 0.06$ & - & $\begin{array}{c}1.76(1.30-2.39) \\
<0.001\end{array}$ \\
\hline $\begin{array}{l}\text { Donor, mismatched } \\
\text { vs. matched }\end{array}$ & $3.43(1.70-6.92), 0.001$ & $3.45(1.72-7.04), 0.001$ & $\begin{array}{l}2.69(1.66-4.37) \\
<0.001\end{array}$ & $\begin{array}{l}2.68(1.65-4.35) \\
<0.001\end{array}$ & $1.21(0.64-2.29), 0.56$ & $1.22(0.65-2.30), 0.54$ & $1.84(0.86-3.93), 0.11$ & $1.89(0.88-4.05), 0.10$ \\
\hline $\begin{array}{l}\text { Stem cell source, } \\
\text { PB vs. BM }\end{array}$ & $0.34(0.12-0.98), 0.05$ & $0.32(0.11-0.94), 0.04$ & $0.74(0.30-1.86), 0.53$ & $0.75(0.30-1.88), 0.54$ & $0.19(0.07-0.54), 0.002$ & $0.19(0.07-0.54), 0.002$ & $0.13(0.04-0.41), 0.001$ & $0.13(0.04-0.42), 0.001$ \\
\hline ATG, yes vs. no & $1.42(0.59-3.41), 0.43$ & $1.45(0.60-3.52), 0.41$ & $1.64(0.95-2.84), 0.08$ & $1.72(1.00-2.97), 0.05$ & $0.96(0.54-1.70), 0.88$ & $0.97(0.55-1.73), 0.93$ & $\begin{array}{l}4.15(1.45-11.89) \\
0.008\end{array}$ & $\begin{array}{l}4.12(1.42-11.92) \\
0.009\end{array}$ \\
\hline $\begin{array}{l}\text { Conditioning, } \\
\text { MAC vs. RIC }\end{array}$ & $1.50(0.53-4.23), 0.45$ & $1.43(0.51-4.07), 0.50$ & $1.36(0.64-2.91), 0.42$ & $1.27(0.60-2.70), 0.54$ & $1.33(0.55-3.24), 0.53$ & $1.27(0.52-3.08), 0.60$ & $2.78(1.00-7.75), 0.05$ & $2.86(1.01-8.07), 0.05$ \\
\hline $\begin{array}{l}\text { Disease stage, high } \\
\text { vs intermediate/low }\end{array}$ & $0.73(0.36-1.47), 0.38$ & $0.69(0.34-1.40), 0.30$ & $1.38(0.88-2.15), 0.16$ & $1.34(0.86-2.09), 0.20$ & $1.52(0.89-2.60), 0.12$ & $1.50(0.88-2.55), 0.14$ & $1.51(0.76-3.03), 0.24$ & $1.44(0.72-2.91), 0.30$ \\
\hline Goodness-of-fit test ${ }^{\dagger}$ & $\begin{array}{c}X^{2}=6.43(8 d f) \\
p=0.60\end{array}$ & $\begin{aligned} X^{2}= & 6.83(8 d f) \\
p & =0.56\end{aligned}$ & $\begin{array}{c}X^{2}=10.10(8 d f) \\
p=0.29\end{array}$ & $\begin{array}{c}X^{2}=11.26(8 d f) \\
p=0.19\end{array}$ & $\begin{aligned} X^{2} & =6.16(8 d f) \\
p & =0.63\end{aligned}$ & $\begin{aligned} X^{2}= & 8.24(8 d f) \\
p & =0.41\end{aligned}$ & $\begin{array}{c}X^{2}=7.47(8 d f) \\
p=0.49\end{array}$ & $\begin{array}{c}X^{2}=10.08(8 d f) \\
p=0.26\end{array}$ \\
\hline
\end{tabular}

Abbreviations: ANC, absolute neutrophil count; aOR, adjusted odds ratio; ATG, antithymocyte globulin; $\mathrm{CI}$, confidence interval; df, degrees of freedom; IL-18, interleukin-18; MAC, myeloablative conditioning; RIC, reduced intensity conditioning. ${ }^{*}$ Each one unit increase in log2 corresponds to a doubling in the corresponding cytokine level. ${ }^{\dagger}$ Hosmer-Lemeshow test [22] 
In the confirmation cohort, higher pre-conditioning levels of both total IL-18 and IL-18BP were associated with increased odds of delayed platelet recovery on day +28 (Table 5). For free IL-18, only a trend towards a higher OR of delayed platelet recovery was observed, whereas no associations with delayed neutrophil recovery were found in univariate analyses (Table 5).

Table 5. Associations of pre-conditioning cytokine serum levels with platelet and neutrophil recovery and corresponding predictive values (confirmation cohort).

\begin{tabular}{|c|c|c|}
\hline \multirow{2}{*}{ Cytokine } & \multicolumn{2}{|c|}{$\begin{array}{l}\text { Odds Ratio (OR) and Area under the ROC Curve (AUC) per log2 Increase * in } \\
\text { Pre-Conditioning Cytokine }\end{array}$} \\
\hline & $\begin{array}{l}\text { Platelets Day }+28 \leq 20 / \mathrm{nL}(n=100) \text { vs. } \\
\qquad 20 / \mathrm{nL}(n=561)\end{array}$ & $\begin{array}{l}\text { ANC Day }+28 \leq 1 / \mathrm{nL}(n=161) \mathrm{vs} \\
\qquad 1 / \mathrm{nL}(n=500)\end{array}$ \\
\hline \multicolumn{3}{|l|}{ Total IL-18 } \\
\hline OR $(95 \% \mathrm{CI}), p$ & 1.42 (1.07-1.90), 0.02 & 1.15 (0.91-1.45), 0.26 \\
\hline $\operatorname{AUC}(95 \% \mathrm{CI}), p$ & $0.58(0.52-0.65), 0.008$ & $0.54(0.49-0.59), 0.13$ \\
\hline \multicolumn{3}{|l|}{ IL-18BP } \\
\hline OR $(95 \%$ CI $), p$ & $2.60(1.45-4.66), 0.001$ & $1.20(0.79-1.82), 0.39$ \\
\hline $\operatorname{AUC}(95 \% \mathrm{CI}), p$ & $0.60(0.54-0.67), 0.001$ & $0.56(0.51-0.61), 0.02$ \\
\hline \multicolumn{3}{|l|}{ Free IL-18 } \\
\hline OR $(95 \% \mathrm{CI}), p$ & $1.31(0.98-1.76), 0.07$ & $1.10(0.86-1.40), 0.46$ \\
\hline $\operatorname{AUC}(95 \% \mathrm{CI}), p$ & $0.56(0.50-0.63), 0.04$ & $0.53(0.48-0.58), 0.28$ \\
\hline \multicolumn{3}{|l|}{ CXCL9 } \\
\hline OR $(95 \% \mathrm{CI}), p$ & $0.90(0.71-1.14), 0.38$ & $0.97(0.81-1.16), 0.70$ \\
\hline $\operatorname{AUC}(95 \% \mathrm{CI}), p$ & $0.45(0.35-0.56), 0.34$ & $0.48(0.40-0.55), 0.53$ \\
\hline \multicolumn{3}{|c|}{$\begin{array}{l}\text { * Each one unit increase in log2 corresponds to a doubling in the corresponding cytokine level. Abbreviations: ANC, } \\
\text { absolute neutrophil count; AUC, area under the ROC curve; CI, confidence interval; CXCL, chemokine (C-X-C motif) } \\
\text { ligand; IFN } \gamma \text {, interferon gamma; IL-18, interleukin-18; IL-18BP, interleukin-18 binding protein; OR, odds ratio; ROC, } \\
\text { receiver operating characteristic. }\end{array}$} \\
\hline \multicolumn{3}{|c|}{$\begin{array}{l}\text { However, after multivariable adjusting for confounders, increasing pre-conditioning levels o } \\
\text { total and free IL-18 correlated with non-achievement of the day }+28 \text { platelet recovery mileston } \\
\text { usted OR } 1.8 \text { and } 1.7 \text {, respectively), whereas the associations with neutrophil recovery did no } \\
\text { h statistical significance (Table } 6) \text {. }\end{array}$} \\
\hline
\end{tabular}


Table 6. Multivariable logistic regression analysis of pre-conditioning total and free IL-18 serum levels with regard to platelet and neutrophil recovery (confirmation cohort).

\begin{tabular}{|c|c|c|c|c|}
\hline \multirow{2}{*}{ Covariate, Effect } & \multicolumn{2}{|c|}{$\begin{array}{c}\text { Platelets Day }+28 \leq 20 / \mathrm{nL}(n=100) \\
\text { Vs. }>20 / \mathrm{nL}(n=561)\end{array}$} & \multicolumn{2}{|c|}{$\begin{array}{c}\text { ANC Day }+28 \leq 1 / \mathrm{nL}(n=161) \\
\text { Vs. }>1 / \mathrm{nL}(n=500)\end{array}$} \\
\hline & $\begin{array}{l}\text { Model with total IL-18, } \\
\text { aOR }(95 \% \mathrm{CI}), p\end{array}$ & $\begin{array}{l}\text { Model with free IL-18, } \\
\text { aOR }(95 \% \text { CI), } p\end{array}$ & $\begin{array}{l}\text { Model with total IL-18, } \\
\quad \text { aOR }(95 \% \text { CI), } p\end{array}$ & $\begin{array}{l}\text { Model with free IL-18, } \\
\text { aOR }(95 \% \text { CI) }, p\end{array}$ \\
\hline Total IL-18, per log2 increase * & $1.80(1.29-2.50),<0.001$ & - & $1.28(0.99-1.65), 0.06$ & - \\
\hline Free IL-18, per $\log 2$ increase * & - & $1.66(1.19-2.31), 0.003$ & - & $1.22(0.94-1.59), 0.14$ \\
\hline Donor, mismatched vs matched & $1.56(0.94-2.57), 0.08$ & $1.56(0.95-2.58), 0.08$ & $0.84(0.54-1.31), 0.44$ & $0.84(0.54-1.32), 0.45$ \\
\hline Stem cell source, $\mathrm{PB}$ vs BM & $0.51(0.24-1.10), 0.09$ & $0.52(0.25-1.11), 0.09$ & $0.19(0.11-0.35),<0.001$ & $0.20(0.11-0.35),<0.001$ \\
\hline ATG, yes vs. no & $3.59(2.04-6.32),<0.001$ & $3.60(2.05-6.33),<0.001$ & $2.96(1.91-4.60),<0.001$ & $2.96(1.90-4.59),<0.001$ \\
\hline Conditioning, MAC vs. RIC & $2.17(1.26-3.76), 0.006$ & $2.18(1.26-3.76), 0.005$ & $1.35(0.85-2.16), 0.20$ & 1.35 (0.85-2.15), 0.21 \\
\hline $\begin{array}{l}\text { Disease stage, high vs. } \\
\text { intermediate/low }\end{array}$ & $2.92(1.70-5.04),<0.001$ & $2.98(1.73-5.13),<0.001$ & $2.06(1.29-3.29), 0.002$ & $2.08(1.30-3.32), 0.002$ \\
\hline Goodness-of- fit test $^{+}$ & $X^{2}=4.84(8 d f), p=0.78$ & $X^{2}=5.31(8 d f), p=0.72$ & $X^{2}=7.91(8 d f), p=0.44$ & $X^{2}=12.35(8 d f), p=0.14$ \\
\hline
\end{tabular}

Abbreviations: ANC, absolute neutrophil count; aOR, adjusted odds ratio; ATG, antithymocyte globulin; CI, confidence interval; df, degrees of freedom; IL-18, interleukin-18; MAC, myeloablative conditioning; RIC, reduced intensity conditioning. ${ }^{*}$ Each one unit increase in $\log 2$ corresponds to a doubling in the corresponding cytokine level. ${ }^{\dagger}$ Hosmer-Lemeshow test [22]. 


\subsection{Day +28 Platelet Recovery and Outcome}

We have previously reported that elevated pre-conditioning IL-18 is a predictor of worse survival due to increased NRM (HR per doubling in IL-18, 1.2-1.4 in both centers) [16].

On univariate analysis, a platelet count $\leq 20 / \mathrm{nL}$ on day +28 was associated with significantly decreased OS within one year post-landmark in both cohorts (HR 1.95 95\%CI 1.22-3.12, $p=0.005$ and HR $2.2595 \%$ CI 1.67-3.03, $p<0.001$, respectively). This was due to markedly increased hazards of NRM (HR 2.13 95\%CI 1.09-4.16, $p=0.03$ and HR 2.94 95\% CI 2.07-4.18, $p<0.001$ in the training and the confirmation cohort, respectively), but not to relapse deaths. The results of the univariable analyses were depicted in Figure 3. The association of the non-achievement of the day +28 platelet recovery milestone with increased NRM in both cohorts could be further substantiated in the corresponding multivariable models (Table S5).
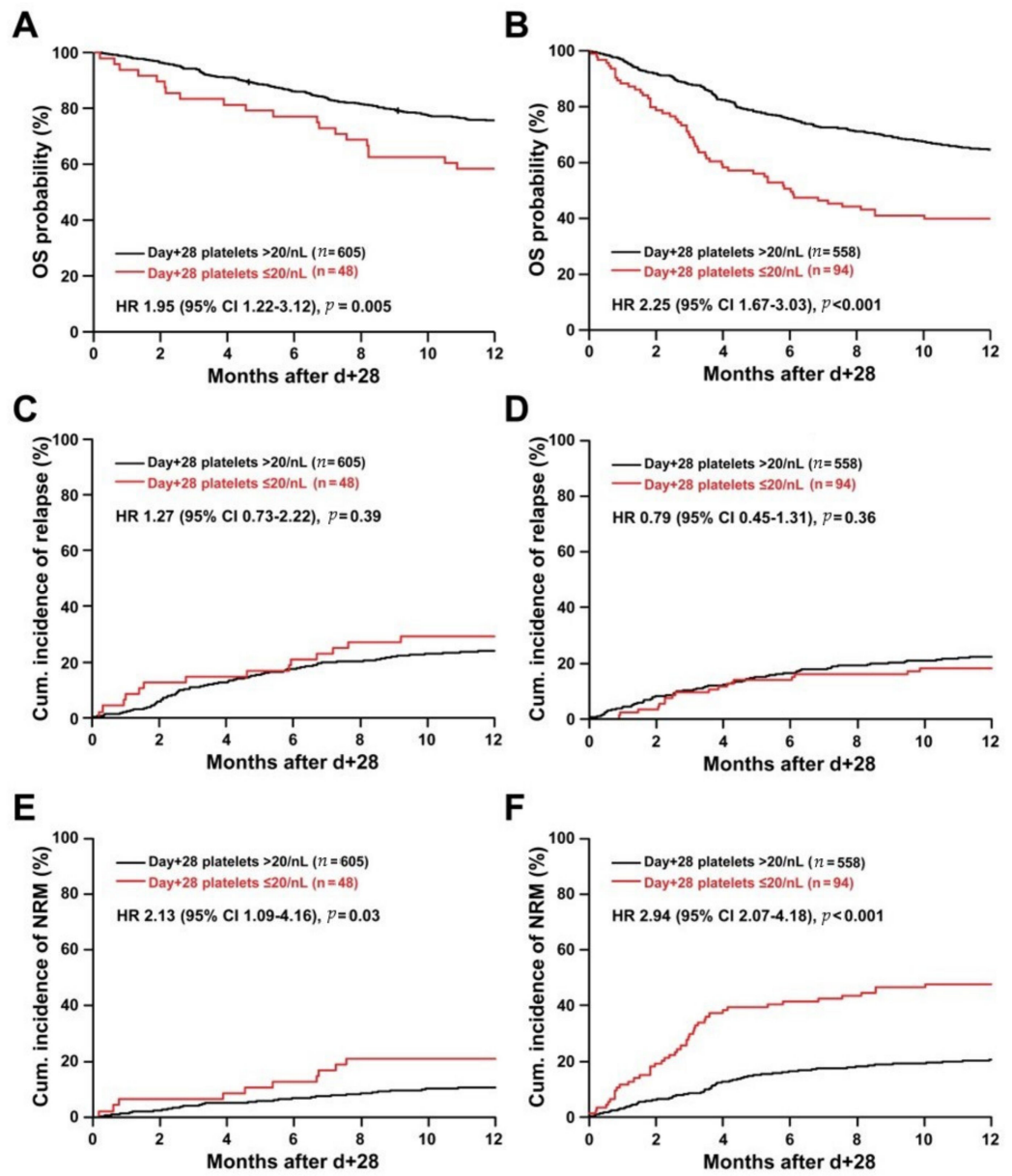

Figure 3. Outcome of allografted patients in the first year post-landmark stratified according to day +28 platelet counts in the training cohort and in confirmation cohort. (A) In the training cohort ( $n=653$ ), platelets $\leq 20 / \mathrm{nL}$ at day +28 were associated with lower probability of overall survival (OS) with the first year after day +28 . (C) Platelets $\leq 20 / n L$ at day +28 were not correlated with incidence of relapse in the training cohort. (E) In contrast, platelets $\leq 20 / \mathrm{nL}$ at day +28 were associated with a significantly increased cumulative incidence of non-relapse mortality (NRM) in the training set. $(\mathbf{B}, \mathbf{D}, \mathbf{F})$ In the confirmation cohort $(n=652)$, a comparable association of platelet counts $<20 / n L$ with worse OS, due to a higher incidence of NRM rather than relapse, was observed. Note: Patients who experienced relapse within the first 28-day post-transplant were excluded from the analysis. Abbreviations: $\mathrm{CI}$, confidence interval; $\mathrm{HR}$, hazard ratio; OS overall survival, NRM, non-relapse mortality. 


\section{Discussion}

The present study demonstrates an association of increased IL-18 serum levels with impaired hematopoietic reconstitution in alloSCT recipients. In particular, high IL-18 correlated with a delayed platelet recovery. In contrast, IFN $\gamma$, or the inflammatory IFN $\gamma$ response chemokines CXCL9 and CXCL10, were not associated with hematopoietic recovery in our study. In light of preclinical evidence that IL-18 is a novel and context-dependent regulator of HSC quiescence [17], our findings may be explained by a direct inhibitory effect of IL-18 on stem cell proliferation in allografted patients.

Affecting up to $27 \%$ of patients in some studies [2], poor graft function is a relevant complication of alloSCT associated with infections and hemorrhages and considerable morbidity and mortality [3]. Both the speed and the quality of post-transplant hematopoietic recovery may be affected by various factors, including disease status at transplant, stem cell source, human leukocyte antigen (HLA) compatibility, donor type, intensity of the conditioning regimen, and the use of in vivo T-cell depletion [3]. Usually, the time to achieve a given neutrophil or platelet count is used as indicator of engraftment/recovery of the recipient's hematopoietic system. However, this parameter does not necessarily reflect the robustness of hematopoietic recovery in the long term after alloSCT. In addition, cytopenias observed following alloSCT may be the result of both slow recovery of blood counts and decreasing blood counts after initially successful engraftment [3].

Delayed platelet recovery following alloSCT is a well-acknowledged risk factor for increased NRM and poor survival [2,5-9]. The underlying mechanism of prolonged thrombocytopenia after alloSCT is complex and may include both impaired thrombopoiesis and increased platelet turnover [23]. Several studies suggest reduced differentiation of megakaryocytes from stem cells and defects in megakaryocytic maturation rather than peripheral destruction of platelets to account for prolonged thrombocytopenia after alloSCT [24,25]. Recently, direct damaging effects of inflammation-associated cytokines on ex vivo megakaryocytic and hematopoietic stem cell function have been demonstrated [26].

In our study, higher levels of pre-conditioning total and free IL-18 were correlated with significantly lower median platelet counts but not ANC, both prior to the start of the conditioning and at various time-points after alloSCT. By comparison, high levels of total and free IL-18 measured at day 0-3 were associated with both lower median platelet counts up to one year after alloSCT and lower median ANC up to day +50 post-transplant. Accordingly, increasing levels of both pre-conditioning and day $0-3$ total and free IL-18 were correlated with the risk of non-achievement of day +28 platelet and ANC recovery milestones. These associations were even stronger for day 0-3 IL-18 levels and were decreasing over time with respect to platelets. Interestingly, no associations of IFN $\gamma$ and the IFN $\gamma$-inducible CXC family chemokines CXCL9 and CXCL10 on platelet and neutrophil recovery were observed. Since IL-18 is an IFN $\gamma$-inducing cytokine and IL-18 and CXC family chemokines show a high level of intercorrelation [16], these findings suggest that myeloid recovery might be affected by IL-18 independently of IFN $\gamma$.

In the context of alloSCT, IL-18 has been investigated primarily with respect to GVHD but clinical studies were often limited by the small numbers of subjects investigated [27-30]. Consequently, a correlation of IL-18 with GVHD severity was found in some alloSCT studies $[27,28]$ but not in others $[29,30]$. We have previously reported that elevated pre-conditioning IL-18 levels predicted worse survival due to increased NRM [16]. In this previous study, median levels of total and free IL-18 in healthy controls were 175 and $141 \mathrm{pg} / \mathrm{mL}$, respectively, which is approximately 4- and 3-fold lower as compared to the median pre-conditioning levels observed in allografted patients [16]. We can only speculate about the underlying causes. In the present study, IL-18 levels were not associated with patient age, patient gender, the HCT-CI and the disease stage prior to alloSCT. However, pre-conditioning total and free IL-18 levels were significantly higher in patients with elevated CRP and ferritin levels consistent with a pro-inflammatory state. Given the implications of the IL-18/IL-18BP pathway in endothelial dysfunction [31,32] and the fact that IL-18 may be released from dying endothelium [11], high IL-18 levels in patients undergoing alloSCT may also reflect endothelial distress caused by the underlying hematologic malignancy and/or the previous antineoplastic treatment. 
IFN $\gamma$-independent effects of IL-18 have gained recent attention [18]. In murine transplant models, BM inflammation involving increased inflammasome activation and enhanced secretion of IL-18 and other cytokines has been demonstrated [33]. Consequently, inhibition of caspase-1 and inflammasome activation was shown to attenuate BM inflammation and promote hematopoietic reconstitution as reflected by increased numbers of megakaryocytes and platelets in transplanted mice. Notably, in their elaborate study on BM niche factors Silberstein and colleagues [17] demonstrated IL-18 as a previously unrecognized quiescence regulator of short-term HSPC. By limiting HSPC proliferation in murine transplant models, IL-18 was able to protect HSPC from 5-FU-induced apoptosis, however, at the expense of a constrained hematopoietic recovery. Similar observations were made by Bordoni et al. [34] in the context of primary HIV infection and the IL-18 stress-response with respect to in vivo proliferation and expansion of lymphoid progenitor stem cells. As regards our observations, the lack of correlation between platelet and neutrophil recovery and levels of IFN $\gamma$ and IFN $\gamma$-inducible chemokines may suggest specific and IFN $\gamma$-independent effects of IL-18 as a regulator of HSPC quiescence in patients undergoing alloSCT. This is of particular relevance, since modulation of IL-18 function is likely to be more effective in conditions with a minor role for IFN $\gamma$ [18].

IL-18 is a known component of the human systemic inflammatory response facilitating T-cell responses and natural killer cell activation [35]. Consequently, high IL-18 levels may also affect post-transplant immune reconstitution. Furthermore, in patients with immune thrombocytopenia, Shan et al. [36,37] demonstrated elevated plasma levels of IL-18 but not IL-18BP during active stages of the disease, proposing also a role for IL-18/IL-18BP imbalance in the pathogenesis and course of immune thrombocytopenia. In our study, we have focused on myeloid reconstitution. Detailed information on post-transplant lymphocyte subset counts was not available. However, it should be noted that pre-conditioning IL-18 levels (i.e., prior to the immune challenge of alloSCT) were also associated with lower pre-conditioning platelet counts. In addition, in the training set, high IL-18 levels measured in the early peri-transplant period (day $0-3$ ), which is characterized by deep aplasia and strong immunosuppressive effects of the starting GVHD-prophylaxis, were also correlated to both delayed platelet and neutrophil recovery. Therefore, although immune effects of IL-18 in the sense of enhanced or abnormal $\mathrm{T}$ cell responses triggering immunity against platelets cannot be ruled out, the associations of high IL-18 with post-transplant myeloid reconstitution are more likely to involve impaired hematopoietic stem cell function.

To the best of our knowledge, in vivo effects of IL-18 on hematologic recovery in allografted patients have not been studied so far. In septic patients, serum concentrations of IL-18 were negatively correlated with platelet counts suggesting a role of IL-18 in the development of severe thrombocytopenia during sepsis [38]. Recently, Korpelainen and colleagues [39] in their study on hematological patients receiving intensive chemotherapy found an inverse correlation between IL-18 and leukocyte counts and an association with complicated courses of febrile neutropenia in the subgroup of autologous stem cell recipients with lymphoma. Consequently, it is tempting to speculate that IL-18 neutralization may represent a means to enhance hematopoietic engraftment and/or reduce occurrence of cytopenias in patients with elevated IL-18 levels.

Among the available inhibitors of IL-18 function, Tadekinig alfa, which is a recombinant human IL-18BP, may represent the most promising agent for IL-18 modulation in terms of post-transplant graft recovery. Tadekinig alfa has been investigated in a phase II, open-label study on patients with adult-onset Still's disease (AOSD) showing a favorable safety profile and early signs of clinical efficacy [40]. In addition, in a recent report, prolonged administration of Tadekinig alfa was safe and shown to be associated with a marked decrease in circulating levels of free IL-18 and improvement of AOSD disease manifestations [41]. The results presented here may provide a rationale for clinical studies exploring the efficacy of Tadekinig alfa in the setting of alloSCT.

Interestingly, platelets themselves may be viewed as versatile intravascular effectors involved in various mechanisms to promote immune responses and are thus increasingly recognized as critical determinants of host defense against infection [42,43]. In septic patients, and similar to IL-18 [14,15], 
thrombocytopenia has been demonstrated as an independent and complementary risk factor for mortality in multiple studies [43]. In our patients, the persistence of thrombocytopenia $\leq 20 / \mathrm{nL}$ beyond day +28 post-transplant in the absence of relapse was a strong predictor of early non-relapse and overall mortality, adding to the existing evidence supporting the adverse prognostic significance of a delayed platelet recovery [2,5-9]. To this end, linking high IL-18 to delayed platelet recovery in allografted patients, the findings presented here may provide an explanation for our previous observation that increased levels of IL-18 are associated with excess NRM due to fatal infectious complications [16].

Obviously, the findings of this study have to be seen in light of some limitations. First, the retrospective and observational design makes the findings susceptible to bias and unknown confounders. Second, the lack of day 0-3 biomarkers levels for the confirmation cohort needs to be acknowledged. Finally, as with all observational studies, we cannot provide evidence for a causal effect of elevated IL-18 levels on hematopoietic recovery after alloSCT, and therefore the results need to be interpreted with caution.

\section{Materials and Methods}

\subsection{Patients}

Adult patients who were allografted between 2004 and 2015 at the University Hospital Heidelberg, Germany, and had provided permission for sample and data collection comprised the training cohort $(n=714)$. In the training cohort, pre-conditioning serum samples (obtained prior to the start of the conditioning regimen) were available for 602 patients, day $0-3$ sera could be retrieved for 306 patients (overlapping patients between pre-conditioning and day $0-3, n=194$ ).

The confirmation cohort consisted of 673 adult patients who underwent alloSCT at the Department of Bone Marrow Transplantation of the University Hospital Essen, Germany, between 2009 and 2013 and had pre-conditioning serum samples available. Written informed consent according to the Declaration of Helsinki was obtained for all patients, and the local ethics committees had approved the sample (ethics committee approval number is 120/2002) and data collection. Patient and outcome data were obtained from medical records and chart review. Disease stage prior to alloSCT and the hematopoietic cell transplantation specific comorbidity index (HCT-CI) was assessed by applying published criteria $[19,44]$.

\subsection{Assessment of Cytokine Serum Levels}

Serum samples were collected in gel tubes and cryopreserved at $-80^{\circ} \mathrm{C}$. Serum levels of total IL-18, IL-18BP, IFN $\gamma$ as well as the IFN $\gamma$-inducible CXC family chemokines CXCL9 and CXCL10 were assessed by ELISA using commercial kits (DuoSet, R\&D Systems, Wiesbaden, Germany) according to the manufacturer's instructions. The concentration of free IL-18 was calculated as described previously [16]. All values were given in $\mathrm{pg} / \mathrm{mL}$.

\subsection{Definitions and Statistical Analysis}

Continuous and categorical variables of patient characteristics were compared using the MannWhitney $\mathrm{U}$ test and the $\chi 2$ test, respectively.

Platelet and absolute neutrophil counts were assessed prior to conditioning and at defined time-points post-transplant (day +14 , day +28 , day +50 , day +100 and year +1 ). The first $(Q 1)$ and fourth quartile (Q4) of the distributions of total IL-18 and free IL-18 were used to stratify patients in low and high IL-18 groups and the median platelet and neutrophil counts were compared with regard to pre-conditioning and day 0-3 levels of total IL-18 and free IL-18. Patients who experienced relapse of the underlying malignancy were excluded from analysis of subsequent landmarks. In addition, correlations between neutrophil and platelet counts were also assessed by employing Spearman's rank correlation coefficients. 
To evaluate associations of cytokines levels with post-transplant hematopoietic recovery, platelet and absolute neutrophil counts (ANC) at defined landmarks were analyzed. For platelets, a delay in recovery was defined as platelets $\leq 20 / \mathrm{nL}$ at day $+28, \leq 50 / \mathrm{nL}$ at day +100 and $\leq 150 / \mathrm{nL}$ at year +1 . For neutrophils, a delay in recovery was defined as ANC $\leq 1 / \mathrm{nL}$ at day +28 .

In absence of established reference ranges, cytokine concentrations were analyzed as continuous variables. Since all cytokine serum levels showed a left-skewed distribution, data were $\log 2$ transformed. Univariate logistic regression was used to evaluate the association of each cytokine with the outcome of interest. Odds ratios (OR) from these models refer to the increase in odds of the outcome for a twofold increase in the corresponding cytokine. In addition, receiver operating characteristic (ROC) curve analysis with calculation of the area under the curve (AUC) was performed. The results were displayed in tables showing OR and AUC estimates together with the 95\% confidence interval (95\% CI) and the $p$ values.

To adjust for known confounders, multivariable logistic regression was used for the effects of total and free IL-18 including donor HLA-matching, stem cell source, anti-thymocyte globulin (ATG) use, type of conditioning, and disease status at transplant as covariates. Model calibration was evaluated by using the Hosmer-Lemeshow goodness-of-fit test [22].

For the association of delayed platelet recovery on day +28 with alloSCT outcome, overall survival (OS), time to relapse, and NRM (death in the absence of prior relapse) within the first year post-landmark were analyzed from day +28 post-transplant to the appropriate endpoint. NRM and recurrence of the underlying malignancy were considered as competing events and cumulative incidence functions were implemented [45]. For multivariable analysis of predictors of OS, NRM and relapse after day +28 , Cox proportional hazard regression models were performed including the covariates specified above and age as an additional confounder [46].

Calculations were done using IBM ${ }^{\circledR}$ SPSS ${ }^{\circledR}$ Statistics, Version 24.0.0 (IBM, Armonk, NY, USA) and the statistical software environment $R$, version 3.3.2 (R Foundation, Vienna, Austria) together with the $\mathrm{R}$ packages 'survplot' version 0.0 .7 , 'rms' version 5.1-2, 'cmprsk' version 2.2-7, 'survival' version 2.42-6. All statistical tests were two-sided. Effects were estimated with $95 \%$ confidence interval $(95 \% \mathrm{CI})$. Results with $p$ values $<0.05$ were considered to be statistically significant.

\section{Conclusions}

The results of the present study demonstrate an association of high IL-18 serum levels with delayed platelet recovery in patients undergoing alloSCT. Given the functions of IL-18 in regulating HSPC quiescence, the mechanisms underlying this association are likely to involve direct and IFN $\gamma$-independent inhibitory effects of IL-18 on hematopoietic progenitor proliferation in allografted patients. The findings may provide a rationale to explore modulation of IL-18 for improving outcomes of alloSCT.

Supplementary Materials: The following are available online at http://www.mdpi.com/2072-6694/12/10/2789/s1. Figure S1: Associations of pre-conditioning total IL-18 serum levels with pre-transplant patient characteristics and pre-conditioning CRP and ferritin levels, Figure S2: Associations of pre-conditioning free IL-18 serum levels with pre-transplant patient characteristics and pre-conditioning CRP and ferritin levels, Figure S3: Associations of day 0-3 total IL-18 serum levels with pre-transplant patient characteristics and pre-conditioning CRP and ferritin levels, Figure S4: Associations of day 0-3 free IL-18 serum levels with pre-transplant patient characteristics and pre-conditioning CRP and ferritin levels, Table S1: Baseline patient characteristics of the day 0-3 cohort, Table S2: Pre-conditioning versus day 0-3 cytokine levels (median, IQR) in the training cohort: (A) all patients, (B) overlap cohort $(n=194)$, Table S3: Correlation (Spearman's rank correlation coefficients) of pre-conditioning and day 0-3 total and free IL-18 serum levels with platelet and neutrophil counts pre-conditioning and at different time-points following alloSCT: training cohort (A), confirmation cohort (B), Table S4: Multivariable logistic regression analysis of day $0-3$ total and day $0-3$ free IL-18 serum levels with regard to platelet and neutrophil recovery (training cohort), Table S5: Multivariable analysis of day +28 platelets and predictors of overall survival (OS), non-relapse mortality (NRM) and relapse within the first year following day +28 after allogeneic stem cell transplantation (complete case analysis).

Author Contributions: Conceptualization, A.R., L.K. and T.L.; methodology, A.R. and L.K.; validation, A.R., L.K. and T.L.; formal analysis, A.R., L.K., R.B., D.W.B., P.D. and T.L.; investigation, A.R., L.K., R.B., C.M.-T., D.W.B., 
P.D. and T.L.; data curation, A.R., L.K. and T.L.; writing-original draft preparation/review and editing, A.R., L.K., R.B., C.M.-T., D.W.B., P.D. and T.L.; visualization, A.R., and T.L.; supervision, T.L.; funding acquisition, T.L. All authors have read and agreed to the published version of the manuscript.

Funding: This work was supported by the Helmholtz-Alliance on Immunotherapy of Cancer, B.L.U.T. e.V. (Weingarten, Germany), and the Wilhelm-Sander-Stiftung (GrantNo. 2008.068.1).

Acknowledgments: The authors wish to acknowledge the great work of the physicians and the nursing staff of both transplant units, and, of course, the patients for making the study possible. The authors also thank Michael Hess and Alexandra Hof for their expert technical assistance and the construction and maintenance of the serum biobank.

Conflicts of Interest: The authors declare no conflict of interest. The funders had no role in the design of the study; in the collection, analyses, or interpretation of data; in the writing of the manuscript, or in the decision to publish the results.

\section{References}

1. Davies, S.M.; Kollman, C.; Anasetti, C.; Antin, J.H.; Gajewski, J.; Casper, J.T.; Nademanee, A.; Noreen, H.; King, R.; Confer, D.; et al. Engraftment and survival after unrelated-donor bone marrow transplantation: A report from the national marrow donor program. Blood 2000, 96, 4096-4102. [CrossRef] [PubMed]

2. Dominietto, A.; Raiola, A.M.; Van Lint, M.T.; Lamparelli, T.; Gualandi, F.; Berisso, G.; Bregante, S.; Frassoni, F.; Casarino, L.; Verdiani, S.; et al. Factors influencing haematological recovery after allogeneic haemopoietic stem cell transplants: Graft-versus-host disease, donor type, cytomegalovirus infections and cell dose. Br. J. Haematol. 2001, 112, 219-227. [CrossRef] [PubMed]

3. Wolff, S.N. Second hematopoietic stem cell transplantation for the treatment of graft failure, graft rejection or relapse after allogeneic transplantation. Bone Marrow Transplant. 2002, 29, 545-552. [CrossRef] [PubMed]

4. LaRocca, A.; Piaggio, G.; Podestà, M.; Pitto, A.; Bruno, B.; Di Grazia, C.; Gualandi, F.; Occhini, D.; Raiola, A.M.; Dominietto, A.; et al. Boost of CD34+-selected peripheral blood cells without further conditioning in patients with poor graft function following allogeneic stem cell transplantation. Haematologica 2006, 91, 935-940. [PubMed]

5. Wagner, J.; Flowers, M.E.; Longton, G.; Storb, R.; Martin, P. Use of screening studies to predict survival among patients who do not have chronic graft-versus-host disease at day 100 after bone marrow transplantation. Biol. Blood Marrow Transplant. 2001, 7, 239-240. [CrossRef]

6. Bolwell, B.; Pohlman, B.; Sobecks, R.; Andresen, S.; Brown, S.; Rybicki, L.; Wentling, V.; Kalaycio, M. Prognostic importance of the platelet count 100 days post allogeneic bone marrow transplant. Bone Marrow Transplant. 2003, 33, 419-423. [CrossRef]

7. Ramírez, P.; Brunstein, C.G.; Miller, B.S.; DeFor, T.; Weisdorf, D. Delayed platelet recovery after allogeneic transplantation: A predictor of increased treatment-related mortality and poorer survival. Bone Marrow Transplant. 2010, 46, 981-986. [CrossRef]

8. Akahoshi, Y.; Kimura, S.-I.; Gomyo, A.; Hayakawa, J.; Tamaki, M.; Harada, N.; Kusuda, M.; Kameda, K.; Ugai, T.; Wada, H.; et al. Delayed platelet recovery after allogeneic hematopoietic stem cell transplantation: Association with chronic graft-versus-host disease and survival outcome. Hematol. Oncol. 2017, 36, 276-284. [CrossRef]

9. Moneib, H.; Hafez, H.; Abdalla, A.; Hassanain, O.; Lehmann, L.; El Haddad, A. Day +100 Platelet Count Predicts Survival After Allogeneic Hematopoietic Stem-Cell Transplantation in Children With Hematologic Malignancies. Clin. Lymphoma Myeloma Leuk. 2019, 19, e221-e227. [CrossRef]

10. Novick, D.; Kim, S.; Kaplanski, G.; Dinarello, C.A. Interleukin-18, more than a Th1 cytokine. Semin. Immunol. 2013, 25, 439-448. [CrossRef]

11. Dinarello, C.A.; Novick, D.; Kim, S.; Kaplanski, G. Interleukin-18 and IL-18 binding protein. Front. Immunol. 2013, 4, 289. [CrossRef] [PubMed]

12. Kaplanski, G. Interleukin-18: Biological properties and role in disease pathogenesis. Immunol. Rev. 2017, 281, 138-153. [CrossRef] [PubMed]

13. Reddy, P.; Ferrara, J.L. Role of interleukin-18 in acute graft-vs-host disease. J. Lab. Clin. Med. 2003, 141, 365-371. [CrossRef]

14. Grobmyer, S.R.; Lin, E.; Lowry, S.F.; Rivadeneira, D.E.; Potter, S.; Barie, P.S.; Nathan, C.F. Elevation of IL-18 in human sepsis. J. Clin. Immunol. 2000, 20, 212-215. [CrossRef] 
15. Emmanuilidis, K.; Weighardt, H.; Matevossian, E.; Heidecke, C.-D.; Ulm, K.; Bartels, H.; Siewert, J.-R.; Holzmann, B. Differential regulation of systemic IL-18 and IL-12 release during postoperative sepsis: High serum IL-18 AS an early predictive indicator of lethal outcome. Shock 2002, 18, 301-305. [CrossRef]

16. Radujkovic, A.; Kordelas, L.; Dai, H.; Schult, D.; Majer-Lauterbach, J.; Dietrich, B.; Müller-Tidow, C.; Dreger, P.; Luft, T. Interleukin-18 and outcome after allogeneic stem cell transplantation: A retrospective cohort study. EBioMedicine 2019, 49, 202-212. [CrossRef]

17. Silberstein, L.; Goncalves, K.A.; Kharchenko, P.V.; Turcotte, R.; Kfoury, Y.; Mercier, F.; Baryawno, N.; Severe, N.; Bachand, J.; Spencer, J.A.; et al. Proximity-Based Differential Single-Cell Analysis of the Niche to Identify Stem/Progenitor Cell Regulators. Cell Stem Cell 2016, 19, 530-543. [CrossRef]

18. Dinarello, C.A.; Kaplanski, G. Indeed, IL-18 is more than an inducer of IFN- $\gamma$. J. Leukoc. Biol. 2018, 104, 237-238. [CrossRef]

19. Gratwohl, A.; Stern, M.; Brand, R.; Apperley, J.; Baldomero, H.; De Witte, T.; Dini, G.; Rocha, V.; Passweg, J.; Sureda, A.; et al. Risk score for outcome after allogeneic hematopoietic stem cell transplantation. Cancer 2009, 115, 4715-4726. [CrossRef]

20. Bacigalupo, A.; Ballen, K.; Rizzo, D.; Giralt, S.; Lazarus, H.; Ho, V.; Apperley, J.; Slavin, S.; Pasquini, M.; Sandmaier, B.M.; et al. Defining the Intensity of Conditioning Regimens: Working Definitions. Biol. Blood Marrow Transplant. 2009, 15, 1628-1633. [CrossRef]

21. Bornhäuser, M.; Kienast, J.; Trenschel, R.; Burchert, A.; Hegenbart, U.; Stadler, M.; Baurmann, H.; Schäfer-Eckart, K.; Holler, E.; Kröger, N.; et al. Reduced-intensity conditioning versus standard conditioning before allogeneic haemopoietic cell transplantation in patients with acute myeloid leukaemia in first complete remission: A prospective, open-label randomised phase 3 trial. Lancet Oncol. 2012, 13, 1035-1044. [CrossRef]

22. Hosmer, D.W.; Lemeshow, S. A goodness-of-fit test for the multiple logistic regression model. Commun. Statist. Theor. Method. 1980, 9, 1043-1069. [CrossRef]

23. Yamazaki, R.; Kuwana, M.; Mori, T.; Okazaki, Y.; Kawakami, Y.; Ikeda, Y.; Okamoto, S. Prolonged thrombocytopenia after allogeneic hematopoietic stem cell transplantation: Associations with impaired platelet production and increased platelet turnover. Bone Marrow Transplant. 2006, 38, 377-384. [CrossRef] [PubMed]

24. Bielski, M.; Yomtovian, R.; Lazarus, H.M.; Rosenthal, N. Prolonged isolated thrombocytopenia after hematopoietic stem cell transplantation: Morphologic correlation. Bone Marrow Transplant. 1998, 22, 1071-1076. [CrossRef] [PubMed]

25. Zhang, X.; Fu, H.; Xu, L.; Liu, D.; Wang, J.; Liu, K.; Huang, X. Prolonged Thrombocytopenia Following Allogeneic Hematopoietic Stem Cell Transplantation and Its Association with a Reduction in Ploidy and an Immaturation of Megakaryocytes. Biol. Blood Marrow Transplant. 2011, 17, 274-280. [CrossRef]

26. Liu, C.; Yang, Y.; Wu, D.; Zhang, W.; Wang, H.; Su, P.; Yao, J.; Liang, C.; Feng, S.; Han, M.; et al. Inflammation-Associated Cytokines IGFBP1 and RANTES Impair the Megakaryocytic Potential of HSCs in PT Patients after Allo-HSCT. Biol. Blood Marrow Transplant. 2018, 24, 1142-1151. [CrossRef]

27. Shaiegan, M.; Iravani, M.; Babaee, G.R.; Ghavamzadeh, A. Effect of IL-18 and sIL2R on aGVHD occurrence after hematopoietic stem cell transplantation in some Iranian patients. Transpl. Immunol. 2006, 15, $223-227$. [CrossRef]

28. Fujimori, Y.; Takatsuka, H.; Takemoto, Y.; Hara,H.; Okamura, H.; Nakanishi, K.; Kakishita, E. Elevated interleukin (IL)-18 levels during acute graft-versus-host disease after allogeneic bone marrow transplantation. Br. J. Haematol. 2000, 109, 652-657. [CrossRef]

29. Scholl, S.; Sayer, H.G.; Kasper, C.; Pietraszczyk, M.; Kliche, K.-O.; Clement, J.H. Increase of interleukin-18 serum levels after engraftment correlates with acute graft-versus-host disease in allogeneic peripheral blood stem cell transplantation. J. Cancer Res. Clin. Oncol. 2004, 130, 704-710. [CrossRef]

30. Nakamura, H.; Komatsu, K.; Ayaki, M.; Kawamoto, S.; Murakami, M.; Uoshima, N.; Yagi, T.; Hasegawa, T.; Yasumi, M.; Karasuno, T.; et al. Serum levels of soluble IL-2 receptor, IL-12, IL-18, and IFN-gamma in patients with acute graft-versus-host disease after allogeneic bone marrow transplantation. J. Allergy Clin. Immunol. 2000, 106, 45-50. [CrossRef]

31. Kahlenberg, J.M.; Thacker, S.G.; Berthier, C.C.; Cohen, C.D.; Kretzler, M.; Kaplan, M. Inflammasome activation of IL-18 results in endothelial progenitor cell dysfunction in systemic lupus erythematosus. J. Immunol. 2011, 187, 6143-6156. [CrossRef] [PubMed] 
32. Durpès, M.-C.; Morin, C.; Paquin-Veillet, J.; Beland, R.; Paré, M.; Guimond, M.-O.; Rekhter, M.; King, G.L.; Geraldes, P. PKC- $\beta$ activation inhibits IL-18-binding protein causing endothelial dysfunction and diabetic atherosclerosis. Cardiovasc. Res. 2015, 106, 303-313. [CrossRef] [PubMed]

33. Qiao, J.; Wu, J.; Li, Y.; Xia, Y.; Chu, P.; Qi, K.; Yan, Z.; Yao, H.; Liu, Y.; Xu, K.; et al. Blockage of caspase-1 activation ameliorates bone marrow inflammation in mice after hematopoietic stem cell transplantation. Clin. Immunol. 2016, 162, 84-90. [CrossRef] [PubMed]

34. Bordoni, V.; Viola, D.; Sacchi, A.; Pinnetti, C.; Casetti, R.; Cimini, E.; Tumino, N.; Antinori, A.; Ammassari, A.; Agrati, C. IL-18 and Stem Cell Factor affect hematopoietic progenitor cells in HIV-infected patients treated during primary HIV infection. Cytokine 2018, 103, 34-37. [CrossRef] [PubMed]

35. Dinarello, C.A.; Fantuzzi, G.; Giamila, F. Interleukin-18 and Host Defense against Infection. J. Infect. Dis. 2003, 187, S370-S384. [CrossRef] [PubMed]

36. Shan, N.-N.; Zhu, X.-J.; Wang, Q.; Wang, C.-Y.; Qin, P.; Peng, J.; Hou, M. High-dose dexamethasone regulates interleukin-18 and interleukin-18 binding protein in idiopathic thrombocytopenic purpura. Haematologica 2009, 94, 1603-1607. [CrossRef] [PubMed]

37. Shan, N.-N.; Zhu, X.-J.; Peng, J.; Qin, P.; Zhuang, X.-W.; Wang, H.-C.; Hou, M. Interleukin 18 and interleukin 18 binding protein in patients with idiopathic thrombocytopenic purpura. Br. J. Haematol. 2009, 144, 755-761. [CrossRef]

38. Zhu, M.; Rong, X.; Li, M.; Wang, S. IL-18 and IL-35 in the serum of patients with sepsis thrombocytopenia and the clinical significance. Exp. Ther. Med. 2019, 19, 1251-1258. [CrossRef]

39. Korpelainen, S.; Hämäläinen, S.; Vänskä, M.; Koivula, I.; Pulkki, K.; Jantunen, E.; Juutilainen, A.; Purhonen, A.-K. Plasma level of interleukin-18 and complicated course of febrile neutropenia in hematological patients after intensive chemotherapy. Cytokine 2020, 129, 155021. [CrossRef]

40. Gabay, C.; Fautrel, B.; Rech, J.; Spertini, F.; Feist, E.; Kötter, I.; Hachulla, E.; Morel, J.; Schaeverbeke, T.; Hamidou, M.A.; et al. Open-label, multicentre, dose-escalating phase II clinical trial on the safety and efficacy of tadekinig alfa (IL-18BP) in adult-onset Still's disease. Ann. Rheum. Dis. 2018, 77, 840-847. [CrossRef]

41. Kiltz, U.; Kiefer, D.; Braun, J.M.; Schiffrin, E.J.; Girard-Guyonvarc', H.C.; Gabay, C. Prolonged treatment with Tadekinig alfa in adult-onset Still's disease. Ann. Rheum. Dis. 2018, 79, e10. [CrossRef] [PubMed]

42. Nicolai, L.; Gaertner, F.; Massberg, S. Platelets in Host Defense: Experimental and Clinical Insights. Trends Immunol. 2019, 40, 922-938. [CrossRef] [PubMed]

43. Bounes, F.V.; Ruiz, S.; Gratacap, M.-P.; Garcia, C.; Payrastre, B.; Minville, V. Platelets Are Critical Key Players in Sepsis. Int. J. Mol. Sci. 2019, 20, 3494. [CrossRef] [PubMed]

44. Sorror, M.L.; Maris, M.B.; Storb, R.; Baron, F.; Sandmaier, B.M.; Maloney, D.G.; Storer, B. Hematopoietic cell transplantation (HCT)-specific comorbidity index: A new tool for risk assessment before allogeneic HCT. Blood 2005, 106, 2912-2919. [CrossRef]

45. Gray, R.J. A Class of KK-Sample Tests for Comparing the Cumulative Incidence of a Competing Risk. Ann. Stat. 1988, 16, 1141-1154. [CrossRef]

46. Scrucca, L.; Santucci, A.; Aversa, F. Regression modeling of competing risk using R: An in depth guide for clinicians. Bone Marrow Transplant. 2010, 45, 1388-1395. [CrossRef]

(C) 2020 by the authors. Licensee MDPI, Basel, Switzerland. This article is an open access article distributed under the terms and conditions of the Creative Commons Attribution (CC BY) license (http://creativecommons.org/licenses/by/4.0/). 